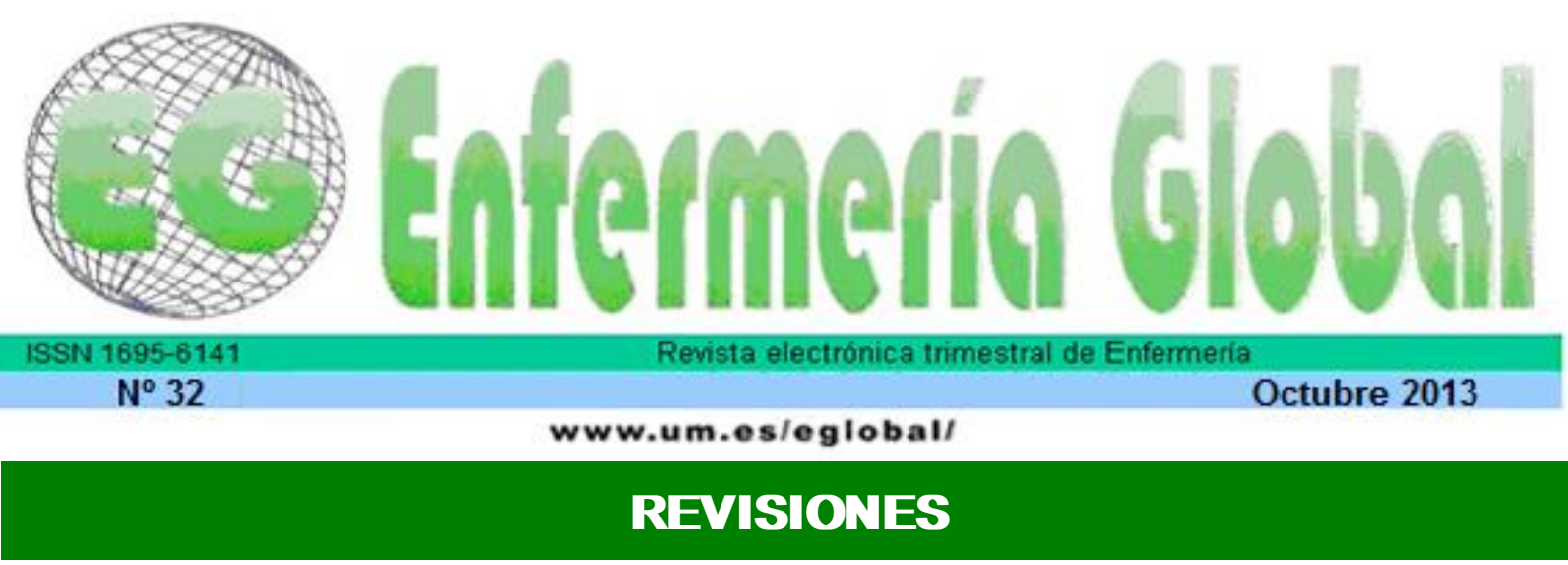

\title{
Traumatismo en pediatría y su relación con los determinantes sociales de la salud
}

Pediatric trauma and its relation to social health determinants

\section{*Pino, Paola Susana *San Juan Hurtado, Lorena Alejandra ${ }^{* *}$ Monasterio Ocares, María Carolina}

\author{
*Enfermera. Hospital Clínico Pontificia Universidad Católica de Chile. E-mail: pspino@uc.cl \\ **Académico Adjunto Escuela de Enfermería Universidad de Chile.
}

\section{Palabras clave: Traumatismo; Pediatría; Determinantes sociales de la salud Keywords: Trauma; Pediatrics; Social heath determinants.}

\section{RESUMEN}

Antecedentes: Los traumatismos representan en Chile y el mundo, la principal causa de muerte en niños, adolescentes y adultos jóvenes, y es una de las causas más importantes de morbilidad. Es un problema relevante de salud pública, debido a que sus consecuencias, son trascendentes en magnitud e impacto.

Objetivo: Identificar los DSS que tienen impacto en el traumatismo en pediatría, describir la relación entre los DSS identificados, y proponer estrategias globales para disminuir el impacto.

Metodología: Revisión bibliográfica, se realizó una búsqueda en las bases de datos Medline/Pubmed, Lilacs, ProQuest, Cinhal y Scielo, entre los meses de octubre 2011 y enero 2012. Para el análisis fueron seleccionados 15 artículos que cumplían con los criterios de inclusión.

Resultados: Los determinantes sociales de la salud que se relacionan con la incidencia y la severidad del traumatismo en pediatría son: educación y literacidad, ingreso y status social, y ambiente físico. Los cuales pueden causar un impacto positivo o negativo en la salud de la población infantil con respecto a la morbimortalidad por causas externas.

Conclusión: Para prevenir el traumatismo infantil en Chile, es indispensable educar a la población. Los programas educativos deben ser diseñados por equipos multidisciplinarios e implementados en forma intersectorial; teniendo en cuenta los determinantes sociales de la salud involucrados, tales como: bajo nivel de escolaridad, bajo nivel socioeconómico y ambiente físico inadecuado; considerando además, la capacidad de las personas para acceder y comprender la información otorgada por los profesionales de la salud. 


\section{ABSTRACT}

Background: Trauma represents in Chile and in the world, the major cause of death in children, adolescents and young adults; and is one of the most important causes of morbidity. It is a significant public health problem, because its consequences are transcendental in magnitude and impact.

Objective: To identify the Social Health Determinants (SHD) that impact trauma in children, describe the relationship between the SHD identified, and propose comprehensive strategies to lessen the impact.

Methods: A literature review was made from Medline/Pubmed, Lilacs, ProQuest, Cinhal and Scielo, between October 2011 and January 2012. Selected for analysis were 15 articles that met the inclusion criteria.

Results: The SHD that are related to the incidence and severity of trauma in children are: education and literacy, socioeconomic status, and physical environment. These can cause a positive or negative impact on the health of children with regard to morbidity and mortality from external causes.

Conclusion: To reduce the trauma of children in Chile, it is essential to educate the population. Educational programs should be designed and implemented by multidisciplinary teams in coordination among sectors, taking into account the social determinants of health involved, such as low educational level, low socioeconomic status and inadequate physical environment; also considering the ability of people to access and understand the information given by health professionals.

\section{INTRODUCCIÓN}

Los traumatismos representan en Chile y el mundo, la principal causa de muerte en niños, adolescentes y adultos jóvenes, y es una de las causas más importantes de morbilidad. Es un problema relevante de salud pública, debido a que sus consecuencias, son trascendentes en magnitud e impacto en cuanto a sufrimiento humano, a los notables costos sociales y económicos para la familia, el estado y las organizaciones de salud privadas, así como a las discapacidades físicas y psicológicas que afectan a la víctima y su entorno ${ }^{1-6}$.

En Chile, las muertes por causas externas constituyen la cuarta causa de muerte en la población general después de las enfermedades del sistema circulatorio, tumores y enfermedades del sistema respiratorio. Durante el año 2009, las causas externas fueron responsables de 8.151 muertes, de los cuales 2.105 fueron accidentes de tránsito, 115 por otros accidentes de trasporte, 2.148 por lesiones autoinflingidas intencionalmente y 3.783 por otras causas externas ${ }^{7}$.

En la población infantil, durante el mismo año, los traumatismos, envenenamientos y algunas otras consecuencias de causas externas (código S00-T98 de la clasificación CIE-10) fueron responsables de 159.653 egresos hospitalarios en niños entre 0 y 4 años, 11.807 en niños entre 5 y 9 años y 9.130 en adolescentes entre 10 y 19 años ${ }^{7}$.

En el Hospital Clínico UC, durante los años 2008 y 2010, se hospitalizaron 398 pacientes menores de 15 años con diagnóstico de traumatismo. De ellos, un 58,8\% fue de sexo masculino, la edad promedio fue 6,5 ( $\pm 4,3$ años). Un 47,2\% ingresó por fractura, $15 \%$ por intoxicación o envenenamiento, 9,3\% por herida cortante, 6,5\% por cuerpo extraño, 2,3\% por quemadura y $20 \%$ por otras lesiones. La estadía hospitalaria fue 2,0 ( \pm 3,9 días). Un 84,4\% tenía Isapre como previsión de salud, $11,6 \%$ Fonasa y $4 \%$ otros (convenios, FFAA, particular). Un $94 \%$ era residente de la 
Zona Centro de Chile, $3,5 \%$ de la Zona Sur y $2,5 \%$ de la Zona Norte (Unidad de Gestión Clínica Red de Salud UC) ${ }^{8}$.

Los objetivos de la presente revisión son: identificar los DSS que tienen impacto en el traumatismo en pediatría, determinar la relación entre los DSS identificados, y proponer estrategias para disminuir el impacto.

El propósito del estudio es contribuir al conocimiento de los profesionales de la salud infantil y sensibilizarlos acerca del importante rol que cumplen en la planificación y desarrollo de estrategias de prevención del traumatismo en pediatría.

\section{METODOLOGÍA}

Para la presente revisión de literatura, se realizó una búsqueda en las bases de datos Medline/Pubmed, Lilacs, ProQuest, Cinhal y Scielo, entre los meses de octubre del año 2011 y marzo del año 2012.

Las palabras claves utilizadas fueron: Trauma; Pediatrics; Children; Risk Factors.

Los criterios de inclusión para la selección de artículos fueron: investigaciones originales y revisiones bibliográficas, en idioma español, portugués e inglés, y con un período de publicación no mayor a 10 años.

Para el análisis fueron seleccionados 15 artículos que cumplían con los criterios de inclusión.

El análisis de los artículos se realizará primero identificando los DSS que tienen impacto en el traumatismo en pediatría, luego determinando la relación los DSS identificados, y finalmente proponiendo estrategias para disminuir el impacto.

\section{Determinantes Sociales de la Salud que tienen impacto en el traumatismo en pediatría.}

Determinantes Sociales de la Salud

Los Determinantes Sociales de Salud son definidos como "aquellas condiciones sociales en las cuales se desarrolla la vida de las personas y que afectan el estado de salud de la población en distinto grado" 9 . Esto alude tanto, a rasgos específicos que afectan al contexto social, como a los mecanismos por los cuales estas condiciones se traducen en impactos a la salud ${ }^{10}$.

En 1996, Health Canadá, desde el paradigma de promoción de la salud, considera importante abordar el conjunto de determinantes de la salud potencialmente modificables, no solamente aquellos que guardan relación con las acciones de los individuos, como los comportamientos y los estilos de vida saludables, sino también, determinantes como el ingreso y estatus social, redes de apoyo social, educación, empleo y condiciones de trabajo, ambiente social, ambiente físico, prácticas personales en salud y destrezas de afrontamiento, desarrollo infantil saludable, biología y dotación genética, servicios de salud, género y cultura ${ }^{11}$. 
El estudio de estos determinantes, cobra vital importancia al momento de visualizar las problemáticas de nuestra población. Tal es el caso de los traumatismos, considerado un importante problema de salud pública dado su magnitud e impacto, constituyen la principal causa de muerte en pacientes pediátricos mayores de 1 año y una de las primeras causas de incapacidad grave en la niñez ${ }^{12-13}$.

Factores de riesgo para la incidencia y severidad del traumatismo en pediatría

Existen estudios internacionales que identifican los factores de riesgo para la incidencia y severidad del traumatismo en la población infantil ${ }^{14-17}$.

En Norteamérica, se identificaron y caracterizaron las áreas con mayores tasas de traumatismo en 9 ciudades y condados de Estados Unidos y Canadá; encontrando que los factores que se asocian a mayores tasas de lesiones graves son: desempleo, menor nivel educacional y bajo nivel socioeconómico ${ }^{14}$.

En Colombia, se estudiaron los factores de riesgo para accidentes en 1.185 niños que consultaron al Policlínico Infantil de Medellín; encontrando que los factores de mayor riesgo son: edad escolar (6 a 10 años), sexo masculino, bajo nivel socioeconómico, desempleo, características de la vivienda como ausencia de protección en escalas y terrazas, y escasa supervisión de los adultos ${ }^{15}$.

En Bolivia, se identificaron los factores de riesgo para accidentes en 600 niños atendidos en un Hospital Pediátrico de La Paz; encontrando que los principales factores de riesgo están relacionados con el trabajo materno fuera del domicilio, y con características de la vivienda como: ausencia de elementos de seguridad (rejas, barandas, protecciones) y presencia de productos peligrosos al alcance de los niños (productos calientes, productos volátiles, objetos cortopunzantes, juegos artificiales, bolsas plásticas, cosméticos, productos de limpieza y medicamentos) ${ }^{16}$.

En España, se analizaron los factores relacionados con la severidad del trauma en 79 niños politraumatizados que ingresaron a un Hospital Universitario en Madrid; encontrando que los factores asociados a mayor severidad del trauma son: los accidentes de tránsito (automovilístico y atropello), y la no utilización de dispositivos de retención en el automóvil ${ }^{17}$.

Según la literatura, los determinantes sociales de la salud que se relacionan con la incidencia y la severidad del traumatismo en pediatría son: educación y literacidad, ingreso y status social, y ambiente físico. Los cuales pueden causar un impacto positivo o negativo en la salud de la población infantil con respecto a la morbimortalidad por causas externas ${ }^{14-17}$.

\section{Relación entre los DSS identificados}

\section{Definición de los DSS identificados}

- Educación y literacidad: La educación, incrementa las elecciones y oportunidades disponibles, incrementa la seguridad y la satisfacción en el trabajo, mejora la "alfabetización en salud", aumenta la seguridad financiera y brinda a las personas las habilidades necesarias para identificar y resolver problemas individuales y grupales ${ }^{11}$. La literacidad en salud, se define como la "habilidad de leer y comprender las 
indicaciones de los medicamentos y otras informaciones relacionadas con el cuidado de la salud, de modo de tener éxito como paciente" ${ }^{18}$.

- Ingreso y status social: El ingreso y el status social alto, actúan como un escudo contra la enfermedad; ya que las personas tienen la capacidad para adquirir vivienda adecuada, alimentos y otras necesidades básicas, para hacer más elecciones y sentirse más en control sobre las decisiones en la vida. Este sentimiento de estar en control es básico para una buena salud ${ }^{11}$.

- Ambiente físico: El ambiente físico afecta la salud directa e indirectamente, ya que una buena salud requiere un medio ambiente sustentable, acceso a buena calidad del agua, aire y alimentos. Así también, son relevantes los factores del ambiente construidos por el hombre como el tipo de vivienda, la seguridad en las comunidades y lugares de trabajo y el diseño vial ${ }^{11}$.

\section{Interrelación entre los DSS identificados}

El nivel de escolaridad que poseen las personas que se encuentran al cuidado de los niños, las condiciones socioeconómicas, y el ambiente físico, son los tres determinantes sociales que influyen en mayor medida en el desarrollo de un accidente ${ }^{19-20}$. La falta de educación en padres y cuidadores, genera un déficit de conocimientos relativos a la prevención de accidentes; además, aumentan su probabilidad de optar a trabajos precarios y con bajos ingresos económicos; lo cual, expone a los infantes, a condiciones de viviendas poco adecuadas para su desarrollo y crecimiento, sobrepobladas y con falta de espacios que contribuyan a resguardar los productos peligrosos ${ }^{19}$.

Identificar los elementos que intervienen, favorecen, predisponen e incluso agravan los accidentes en la infancia, es de vital importancia al momento de elaborar estrategias preventivas ${ }^{19}$. Por este motivo, los esfuerzos preventivos deben ir enfocados principalmente a estimular y desarrollar conductas tendientes a mejorar los conocimientos y cambiar actitudes en el núcleo familiar.

\section{Estrategias para disminuir el impacto}

Existen estrategias para disminuir el impacto del traumatismo en pediatría a nivel internacional y nacional ${ }^{4-5,21-22}$.

A nivel internacional en Estados Unidos se han aplicado con éxito programas de intervención que muestran una importante disminución de la mortalidad por esta causa, en un 30\% aproximadamente, aplicando determinados modelos preventivos. Por ejemplo, el uso de sillas apropiadas en diseño y colocación, para niños pequeños en vehículos que los transportan, ha incidido en una disminución estimada de la letalidad en $69 \%$ entre los lactantes y de $47 \%$ en los niños mayores ${ }^{5}$.

En Argentina, diseñaron un programa educativo, denominado estrategia VAPLE (Vacunas para Prevención de Lesiones), haciendo coincidir las intervenciones educativas con el cronograma del Plan Nacional de Vacunación. Este programa se inicia con el alta de los recién nacidos y se extiende a todas las etapas de la infancia y adolescencia. Ellos concluyeron que la metodología utilizada contribuye a mejorar los conocimientos y comportamientos de la población con respecto a la prevención de accidentes. Las áreas que presentaron mayores dificultades se relacionaron con 
creencias erróneas respecto a vigilancia de los niños y comportamientos inseguros en cuanto a la protección de accidentes eléctricos y uso de sillas de seguridad para autos $^{22}$.

A nivel nacional en Chile, las intervenciones para la prevención de traumatismos, se agrupan en tres grandes grupos: promover cambios por medio de la educación, persuasión, o protección en los niños menores; impulsar las modificaciones en los ambientes de riesgo y estimular las modificaciones de las estructuras e ingeniería involucradas; y disponer las leyes que refuercen las medidas preventivas, disminuyendo los riesgos, vigilando que leyes o reglamentos sean cumplidos y/o que los infractores sean sancionados, según corresponda ${ }^{5}$.

En los objetivos sanitarios para el período 2000-2010, el Ministerio de Salud incluye aumentar la cobertura del tratamiento en unidades de cuidados intensivos de los pacientes politraumatizados, además de implementar programas intersectoriales de prevención de traumatismos y envenenamientos. Además, a través del plan de garantías GES, se espera poder garantizar a los pacientes politraumatizados un manejo de cuidados intensivos integral, atención oportuna y de calidad, que incluya las prestaciones de efectividad demostrada y sea provista por profesionales e instituciones calificados ${ }^{4}$.

Una de las áreas temáticas en el Plan Nacional de Salud 2011-2020 para el cumplimiento de los Objetivos Sanitarios es prevenir y reducir la morbilidad, discapacidad y mortalidad prematura por afecciones crónicas no transmisibles, trastornos mentales, violencia y traumatismos ${ }^{21}$.

\section{CONCLUSIÓN}

Con la presente revisión de literatura, podemos concluir que, para prevenir el traumatismo infantil en Chile, es indispensable educar a la población. Sin embargo, además es necesario regular otras áreas entre las que se encuentran: la utilización de los recursos de ingeniería y diseño de las calles; el control y la vigilancia de la seguridad ambiental; y las formulaciones legislativas y judiciales pertinentes para brindar la protección necesaria a la población infantil ${ }^{5}$.

Los profesionales de la salud son responsables de estimular y desarrollar conductas tendentes a mejorar los conocimientos, cambiar las actitudes, prácticas y estilos de vida tanto en nuestros pacientes como en sus familias; teniendo en cuenta los distintos factores de riesgo presentes en los niños de acuerdo a su sexo, edad y desarrollo psicomotor.

Para lograrlo, los programas educativos deben ser diseñados por equipos multidisciplinarios e implementados en forma intersectorial, teniendo en cuenta los determinantes sociales de la salud involucrados, tales como: bajo nivel de escolaridad, bajo nivel socioeconómico y ambiente físico inadecuado; considerando además, la capacidad de las personas para acceder y comprender la información otorgada por los profesionales de la salud.

Estos programas educativos deben implementarse desde el control prenatal, para realizar un diagnóstico de los padres, evaluando su nivel socioeconómico, conocimientos frente al tema y capacidades de autocuidado; con la finalidad de saber 
si éstos, se encuentran en condiciones de adoptar las medidas de prevención de traumatismos antes del nacimiento.

Las medidas de prevención deben ser adecuadas para el nivel socioeconómico de la familia, para que la falta de recursos no sea un obstáculo; por lo mismo se debería legislar acerca de los precios para las sillas de seguridad, las cuales son bastantes costosas, pero con un impacto muy significativo en la disminución de lesiones graves frente a una colisión ${ }^{5}$.

Cabe destacar la importancia que tienen los medios de comunicación como fuente de información, por lo cual es apropiado utilizar este recurso para difundir las medidas de prevención de traumatismos en forma masiva.

\section{BIBLIOGRAFÍA}

1. García-Huidobro D, Munita $P$, Legarraga $P$, Valenzuela $P$, Cano $C$, Bedregal $P$, et. al. Accidentes en pediatría: oportunidades para la prevención. Rev Méd Chil. [Internet]. 2005 Nov. [cited 2011 Dic 20]; 133 (11), 1389-1391. Available from: http://www.scielo.cl/pdf/rmc/v133n11/art16.pdf

2. García Gariglio L, Gándaro P, Cardozo N, Bianchi M, Santoro A, Pais T, Rubio I. Conceptos, actitudes y prácticas en salud sobre prevención de lesiones en niños menores de 10 años. Arch Pediatr Urug. [Internet]. 2008. [cited 2011 Dic 20]; 79 (4), 284-290. Available from: http://www.scielo.edu.uy/pdf/adp/v79n4/v79n4a03.pdf

3. Iñón $A$, Dimarco $V$, Nasta $C$, Ubeda $C$, Vilar de Saráchaga D. Prevención de lesiones no intencionales: Opiniones y actitudes de los pediatras. Arch Argent Pediatr. [Internet]. 2006 Jan-Feb. [cited 2011 Dic 26]; 104 (1), 64-68. Available from: http://www.scielo.org.ar/pdf/aap/v104n1/v104n1a12.pdf

4. Red salud [Internet]. Chile: Ministerio de Salud; c2007 [cited 2011 Nov 7]. Available from: http://www.redsalud.gov.cl/archivos/guiasges/politraumatizado.pdf

5. Romero P. Accidentes en la infancia: Su prevención, tarea prioritaria en este milenio. Rev Chil Pediatr. [Internet]. 2007 Oct. [cited 2011 Dic 26]; 78 (1), 57-73. Available from: http://www.scielo.cl/pdf/rcp/v78s1/art05.pdf

6. Valenzuela P, Paris E. Manual de Pediatría: Prevención de Accidentes [Internet]. Chile: Editorial Pontificia Universidad Católica de Chile; sf. Chapter 2, Prevención de Accidentes; [cited 2011 Nov 7]. Available from: http://escuela.med.puc.cl/paginas/publicaciones/ManualPed/PrevenAccid.html

7. Instituto Nacional de Estadística: Ministerio de Salud [Internet]. Chile. c2009 - [cited 2011 Nov 7]. Available from: http://deis.minsal.cl/deis/indicadores/IBS Chile 2009.pdf

8. Sistema de información de análisis de casuística hospitalaria. Unidad Gestión Clínica. Red Salud UC.

9. Jadue L, Marín F. Determinantes sociales de la salud en Chile, En la perspectiva de la equidad. 1rs (ed). Chile: ICES; c2005. Chapter 1, Equidad y Determinantes sociales en salud; 9-17.

10. Marmot M. Economic and social determinants of disease. Int $\mathrm{J}$ Epidemiol. [Internet]. 2001 [cited 2011 Nov 7]; 10 (1), 988-989. Available from: http://www.ncbi.nlm.nih.gov/pmc/articles/PMC2566682/pdf/11693982.pdf

11. Moiso A. Fundamentos de salud pública. 1rs ed. La Plata; c2007. Chapter 6, Determinantes de la Salud: p. 686.

12. Pérez C, Buffet C. Manejo Inicial del Trauma Infantil. Rev Ped Elec. [Internet]. 2008. [cited 2012 Jun 12]; 5 (1), 20-31. Avaliable from: www.revistapediatria.cl/vol5num1/pdf/4 TRAUMA.pdf 
13. Nájera G, Galicia R, Morales C, Monterrosas M, Martínez P. Conducta de salud en niño con traumatismo craneoencefálico. Enf Neurol. [Internet]. 2011 [cited 2012 Jun 12]; 10 (2), 72-76. Avaliable from: www.medigraphic.com/pdfs/enfneu/ene2011/ene112d.pdf

14. Newgard C, Schmicker R, Sopko G, Andrusiek D, Bialkowski W, Craig D, et al. Trauma in the Neighborhood: A Geospatial Analysis and Assessment of Social Determinants of Major Injury in North America. Am J Public Health. [Internet]. 2011 Jul [cited 2012 Jun 12]; 101 (4), 669-677. Avaliable from: http://www.ncbi.nlm.nih.gov/pubmed/21389292

15. Salazar O, Medina D, Neira C, Ramírez H, Correa J, Mesa M. Factores de riesgo de accidentes en niños que consultan al Policlínico Infantil de Medellín diciembre 1 de 1998- marzo 6 de 1999. IAETRIA. . [Internet]. 2011 Jun [cited 2012 Jun 12]; 14 (2), 122-131. Avaliable from: http://revinut.udea.edu.co/index.php/piatreia/article/viewFile/3798/3514

16. Paulsen $\mathrm{K}$, Mejía H. Factores de riesgo para accidentes en niños hospital del niño "Dr.

Ovidio Aliaga Uria”. Rev Chil Pediatr. [Internet]. 2005 Jun [cited 2012 Jun 12]; 76 (1), 65-74. Available from: http://www.scielo.cl/scielo.php?pid=S037041062005000100014\&script=sci arttext

17. De Tomás E, Navascués J, Soleto J, Sánchez R, Romero R, García-Casillas M, et al. Factores relacionados con la severidad en el niño politraumatizado. Cir Pediátr. [Internet]. 2004 [cited 2012 Jun 12]; 17: 40-44. Available from: http://www.secipe.org/coldata/upload/revista/2004;17.40-44(1).pdf

18. Medwave [Internet]. Estados Unidos; c2009 [cited 2012 Jun 12]; Available from: http://www.mednet.cl/link.cgi/Medwave/Enfermeria/3921

19. Zayas R, Cabrera U, Simón D. ¿Accidentes Infantiles o lesiones no intencionales?. Rev Cub Peditatr. [Internet]. 2007 [cited 2012 Jun 12]; 79 (1): 1-7. Available from: http://bvs.sld.cu/revistas/ped/vol79_01_07/ped09107.pdf

20.Pérez L. Factores de riesgo para accidentes domésticos en lactantes y prescolares, Hospital Pediátrico "Dr. Agustín Zubillaga". [Internet]. Venezuela: Universidad Centroccidental Lisandro Alvarado; c2003 [cited 2012 Jun 12]. 63p. Available from: http://bibmed.ucla.edu.ve/DB/bmucla/edocs/textocompleto/TWA288P472003.pdf

21. Red salud [Internet]. Chile: Ministerio de Salud; sf. [cited 2011 Nov 7]. Available from:

http://www.redsalud.gov.cl/portal/url/page/minsalcl/g temas/g plan nacional desal ud/plan nacional desalud 2.html

22. Waisman I, Rodríguez M, Malamud B, Zabala R, Echegaray L, Bornoroni G. Un proyecto para prevención de accidentes desde el consultorio del pediatra. Arch Argent Pediatr. [Internet]. 2005 [cited 2012 Jun 12]; 103 (1): 23-38. Available from: http://www.scielo.org.ar/pdf/aap/v103n1/v103n1a06.pdf

ISSN 1695-6141

(C) COPYRIGHT Servicio de Publicaciones - Universidad de Murcia 